\title{
The colored symmetric and exterior algebras
}

\author{
Rafael S. González D’León ${ }^{1} \|^{\dagger}$ \\ ${ }^{1}$ University of Kentucky, Lexington, KY 40506
}

\begin{abstract}
In this extended abstract we present colored generalizations of the symmetric algebra and its Koszul dual, the exterior algebra. The symmetric group $\mathfrak{S}_{n}$ acts on the multilinear components of these algebras. While $\mathfrak{S}_{n}$ acts trivially on the multilinear components of the colored symmetric algebra, we use poset topology techniques to describe the representation on its Koszul dual. We introduce an $\mathfrak{S}_{n}$-poset of weighted subsets that we call the weighted boolean algebra and we prove that the multilinear components of the colored exterior algebra are $\mathfrak{S}_{n^{-}}$ isomorphic to the top cohomology modules of its maximal intervals. We show that the two colored Koszul dual algebras are Koszul in the sense of Priddy et al.

Résumé. Dans ce résumé détaillé, nous présentons des généralisations colorées de l'algèbre symétrique et de sa duale de Koszul, l'algèbre extérieure. Le groupe symétrique $\mathfrak{S}_{n}$ agit sur les composantes multilinéaires de ces algèbres. Tandis que $\mathfrak{S}_{n}$ agit trivialement sur les composantes multilinéaires de l'algèbre symétrique colorée, nous utilisons les techniques topologiques de la théorie des ensembles partiellement ordonnés pour décrire la représentation sur sa duale de Koszul. Nous introduisons un $\mathfrak{S}_{n}$-ensemble partiellement ordonné de sous-ensembles pondérés que nous appelons l'algèbre de Boole pondérée et nous montrons que les composantes multilinéaires de l'algèbre extérieure colorée sont $\mathfrak{S}_{n}$-isomorphes aux modules cohomologiques supérieurs de ses intervalles maximaux. Nous montrons que les deux algèbres colorées sont des Koszul dans le sens apporté par Priddy et al.
\end{abstract}

Keywords. symmetric algebra, exterior algebra, Koszul duality, boolean algebra, poset cohomology, symmetric functions

\section{Introduction}

Let $\mathbf{k}$ denote a field of characteristic not equal to 2 and $V$ be a finite dimensional $\mathbf{k}$-vector space. The tensor algebra $T(V)=\bigoplus_{n \geq 0} V^{\otimes n}$ is the free associative algebra generated by $V$, where $V^{\otimes n}$ denotes the tensor product of $n$ copies of $V$ and $V^{\otimes 0}:=\mathbf{k}$. For any set $R \subseteq T(V)$ denote by $\langle R\rangle$ the ideal of $T(V)$ that $R$ generates. Let $R_{1}$ be the subspace of $V \otimes V$ generated by relations of the form

$$
x \otimes y-y \otimes x \quad \text { (symmetry), }
$$

for all $x, y \in V$. The symmetric algebra $\mathcal{S}(V)$ is the quotient algebra $\mathcal{S}(V):=T(V) /\left\langle R_{1}\right\rangle$.

Now let $R_{2} \subseteq V \otimes V$ be generated by relations of the form

$$
x \otimes y+y \otimes x \quad \text { (antisymmetry), }
$$

\footnotetext{
$\dagger^{\dagger}$ Email: rafaeldleon@uky . edu

1365-8050 @ 2016 Discrete Mathematics and Theoretical Computer Science (DMTCS), Nancy, France
} 
for all $x, y \in V$. The exterior algebra $\Lambda(V)$ is the algebra $\Lambda(V)=T(V) /\left\langle R_{2}\right\rangle$. We will use the concatenation $x y$ to denote the image of $x \otimes y$ in $\mathcal{S}(V)$ and the wedge $x \wedge y$ to denote the image of $x \otimes y$ in $\Lambda(V)$ under the canonical epimorphisms.

Let $V^{*}:=\operatorname{Hom}(V, \mathbf{k})$ denote the vector space dual to $V$. For finite dimensional $V$ we have that $V^{*} \simeq V$. Recall that for an associative algebra $A=A(V, R):=T(V) /\langle R\rangle$ generated on a finite dimensional vector space $V$ and (quadratic) relations $R \subseteq V^{\otimes 2}$ there is another algebra $A^{!}$associated to $A$ that is called the Koszul dual associative algebra to $A$. Indeed, when $V$ is finite dimensional, there is a canonical isomorphism $\left(V^{\otimes 2}\right)^{*} \simeq V^{*} \otimes V^{*}$ and we let $R^{\perp}$ be the image under this isomorphism of the space of elements in $\left(V^{\otimes 2}\right)^{*}$ that vanish on $R$. The Koszul dual $A^{!}$of $A$ is the algebra $A^{!}:=$ $A\left(V^{*}, R^{\perp}\right)=T\left(V^{*}\right) /\left\langle R^{\perp}\right\rangle$. It follows from relations 1.1$]$ and 1.2 that $\Lambda\left(V^{*}\right)$ is the Koszul dual associative algebra to $\mathcal{S}(V)$.

Denote $[n]:=\{1,2, \ldots, n\}$ and let $V=\mathbf{k}[n]$ be the vector space with generators $[n]$. We define the multilinear component $\mathcal{S}(n)$ as the subspace of $\mathcal{S}(V)$ generated by products of the form $\sigma(1) \sigma(2) \cdots \sigma(n)$ where $\sigma$ is a permutation in the symmetric group $\mathfrak{S}_{n}$. Similarly $\Lambda(n)$ is defined to be the subspace of $\Lambda(V)$ generated by wedged permutations, i.e., the generators are of the form $\sigma(1) \wedge \sigma(2) \wedge \cdots \wedge \sigma(n)$ for $\sigma \in \mathfrak{S}_{n}$. The symmetric group acts on the generators of $\mathcal{S}(n)$ and $\Lambda(n)$ by permuting their letters and this action induces representations of $\mathfrak{S}_{n}$ in both $\mathcal{S}(n)$ and $\Lambda(n)$. Using the relations $(1.1)$ and $(1.2)$ we can see that both $\mathcal{S}(n)$ and $\Lambda(n)$ are always one-dimensional spaces with bases given by $\{12 \cdots n\}$ and $\{1 \wedge 2 \wedge \cdots \wedge n\}$ respectively. Moreover, for $n \geq 1$ it is easy to see that

$$
\mathcal{S}(n) \cong_{\mathfrak{S}_{n}} \mathbf{1}_{n} \text { and } \Lambda(n) \cong_{\mathfrak{S}_{n}} \operatorname{sgn}_{n},
$$

where $\mathbf{1}_{n}$ and $\operatorname{sgn}_{n}$ are respectively the trivial and the sign representations of $\mathfrak{S}_{n}$.

\subsection{Colored symmetric and exterior algebras}

Let $\mathbb{N}$ denote the set of nonnegative integers and $\mathbb{P}$ the set of positive integers. For a subset $S \subseteq \mathbb{P}$ we consider the set $[n]^{S}:=[n] \times S$ of colored letters of the form $(x, i)$ (that we will denote $x^{i}$ ) where $x \in[n]$ and $i \in S$. Let $V=\mathbf{k}[n]$ and $V^{S}=\mathbf{k}[n]^{S}$ where $S \subseteq \mathbb{P}$ is finite and $C R_{1} \subseteq V^{S} \otimes V^{S}$ be generated by

$$
\begin{array}{ll}
x^{i} \otimes y^{j}-y^{i} \otimes x^{j} & \text { (label symmetry) }, \\
x^{i} \otimes y^{j}-x^{j} \otimes y^{i} & \text { (color symmetry) }
\end{array}
$$

for all $x, y \in[n]$ and $i, j \in S$. The $S$-colored symmetric algebra $\mathcal{S}_{S}(V)$ is defined to be the algebra $\mathcal{S}_{S}(V):=T\left(V^{S}\right) /\left\langle C R_{1}\right\rangle$. We define the $S$-colored exterior algebra $\Lambda_{S}\left(V^{*}\right)$ on $V^{*}$ as the Koszul dual to $\mathcal{S}_{S}(V)$. Explicitly, the reader can check that if we let $C R_{2} \subseteq V^{S} \otimes V^{S}$ be generated by

$$
\begin{gathered}
x^{i} \otimes y^{i}+y^{i} \otimes x^{i} \quad \text { (monochromatic antisymmetry), } \\
x^{i} \otimes y^{j}+y^{i} \otimes x^{j}+y^{j} \otimes x^{i}+x^{j} \otimes y^{i} \quad \text { (mixed antisymmetry), }
\end{gathered}
$$

for all $x, y \in[n]$ and $i, j \in S$, then $\Lambda_{S}(V)=T\left(V^{S}\right) /\left\langle C R_{2}\right\rangle$. Choosing $S=[k]$ for some $k \in \mathbb{P}$ and letting $k$ be large we obtain the colored symmetric algebra $\mathcal{S}_{\mathbb{P}}(V)$ and the colored exterior algebra $\Lambda_{\mathbb{P}}(V)$. We denote $\mathcal{S}_{\mathbb{P}}(n)$ and $\Lambda_{\mathbb{P}}(n)$ respectively the components of $\mathcal{S}_{\mathbb{P}}(V)$ and $\Lambda_{\mathbb{P}}(V)$ generated by colored permutations and wedged colored permutations. A colored permutation is a permutation $\sigma \in \mathfrak{S}_{n}$ together with a function that assigns to each $x \in[n]$ a color $\operatorname{color}(x) \in \mathbb{P}$. For example $2^{1} 1^{4} 3^{2}$ is a colored 
permutation of [3] (here $\operatorname{color}(1)=4, \operatorname{color}(2)=1$ and $\operatorname{color}(3)=2)$ and hence a generator in $\mathcal{S}_{\mathbb{P}}(3)$. For a colored permutation $\sigma$ of $n$ let $\wedge(\sigma)$ denote the wedged colored permutation $\sigma(1) \wedge \sigma(2) \wedge \cdots \wedge \sigma(n)$. For example $\wedge\left(2^{1} 1^{4} 3^{2}\right)=2^{1} \wedge 1^{4} \wedge 3^{2}$ is a generator in $\Lambda_{\mathbb{P}}(3)$. Let $\mathfrak{S}_{n}^{S}$ denote the set of colored permutations of $[n]$ with colors in $S \subseteq \mathbb{P}$.

A weak composition $\mu$ of $n$ is a sequence of nonnegative integers $(\mu(1), \mu(2), \ldots)$ such that $|\mu|:=$ $\sum_{i \geq 1} \mu(i)=n$. Let wcomp be the set of weak compositions and wcomp w $_{n}$ the set of weak compositions of $n$. For example, $(0,1,2,0,1):=(0,1,2,0,1,0,0, \ldots)$ is in wcomp $_{4}$.

For $\sigma \in \mathfrak{S}_{n}^{\mathbb{P}}$ let $\mu(\sigma) \in$ wcomp be such that $\mu(\sigma)(j)$ is the number of letters of color $j$ in $\sigma$ for each $j$, we call $\mu(\sigma)$ the content of $\sigma$. For $\mu \in$ wcomp we denote by $\mathfrak{S}_{\mu}$ the set of colored permutations of content $\mu$. Define $\mathcal{S}(\mu)$ and $\Lambda(\mu)$ to be respectively the subspaces of $\mathcal{S}_{\mathbb{P}}(|\mu|)$ and $\Lambda_{\mathbb{P}}(|\mu|)$ generated by colored permutations and wedged colored permutations in $\mathfrak{S}_{\mu}$. For example $\mathcal{S}(0,1,2,0,1)$ and $\Lambda(0,1,2,0,1)$ have generators associated with colored permutations of [4] that contain one letter of color 2, two letters of color 3, one letter of color 5 and no other letters. The symmetric group $\mathfrak{S}_{n}$ acts on $\mathcal{S}_{\mathbb{P}}(n)$ and $\Lambda_{\mathbb{P}}(n)$ as before. A permutation $\tau \in \mathfrak{S}_{n}$ acts by replacing the colored letter $x^{i}$ for the colored letter $\tau(x)^{i}$. This action preserves the colors of the generators and so $\mathcal{S}(\mu)$ and $\Lambda(\mu)$ are also representations of $\mathfrak{S}_{n}$. Clearly if $\nu$ is a rearrangement of $\mu$, we have that $\mathcal{S}(\nu) \simeq_{\mathfrak{S}_{n}} \mathcal{S}(\mu)$ and $\Lambda(\nu) \simeq_{\mathfrak{S}_{n}} \Lambda(\mu)$. In particular, if $\mu$ has a single nonzero component then $\mathcal{S}(\mu)$ is isomorphic to $\mathcal{S}(n)$ and $\Lambda(\mu)$ is isomorphic to $\Lambda(n)$.

For $\mu \in$ wcomp $_{n}$ define its support $\operatorname{supp}(\mu)=\{j \in \mathbb{P} \mid \mu(j) \neq 0\}$. Then for $S \subseteq \mathbb{P}$ we have

$$
\mathcal{S}_{S}(n) \simeq_{\mathfrak{S}_{n}} \bigoplus_{\substack{\mu \in \in_{\operatorname{wcomp}} \\ \operatorname{supp}(\mu) \subseteq S}} \mathcal{S}(\mu) \quad \text { and } \quad \Lambda_{S}(n) \simeq_{\mathfrak{S}_{n}} \bigoplus_{\substack{\mu \in \operatorname{wcomp}_{n} \\ \operatorname{supp}(\mu) \subseteq S}} \Lambda(\mu) .
$$

The following theorem follows immediately from relations $[1.3$ and $(1.4)$.

Theorem 1.1 For $n \geq 1$ and $\mu \in \operatorname{wcomp}_{n}$,

$$
\mathcal{S}(\mu) \cong_{\mathfrak{S}_{n}} \mathbf{1}_{n}
$$

Our goal is to understand the more interesting representation of $\mathfrak{S}_{n}$ on $\Lambda(\mu)$ for all $\mu \in$ wcomp. For this we are going to apply the program started by Hanlon and Wachs in [6] and by Wachs in [13]. They applied poset topology techniques to the partially ordered set (or poset) $\Pi_{n}$ of partitions of the set $[n]$, and to the induced subposet of $\Pi_{n}$ where all partitions have parts of size congruent to $1 \bmod (k-1)$, in order to understand algebraic properties of the multilinear components of the free Lie algebra and the free Lie $k$ algebra. The author and Wachs [5] and the author [4] have applied similar techniques to a family of posets of weighted partitions in their study of the operad of Lie algebras with multiple compatible brackets and its Koszul dual operad, the operad of commutative algebras with multiple totally commutative products. The original motivation for the present work is precisely the study of analogous constructions within the category of connected graded associative algebras.

The main idea of the technique in [6, 13, 5, 4] is that in order to study the representation of $\mathfrak{S}_{n}$ on the multilinear component $A(n)$ of certain algebra $A$, a certain poset $P_{A}$ is constructed so that the (co)homology of $P_{A}$ (defined later) is $\mathfrak{S}_{n}$-isomorphic to $A(n)$ (perhaps up to tensoring with the sign representation). Then poset topology techniques applied to $P_{A}$ can recover algebraic information about $A(n)$. We will be following very closely the thread of ideas in [4]. We will recall here some of the concepts involved while referring the reader to consult [4] for most of the background and related notation. 
To every poset $P$ one can associate a simplicial complex $\Delta(P)$ (called the order complex) whose faces are the chains (totally ordered subsets) of $P$. If there is a group $G$ acting on the poset in such a way that every $g \in G$ is a (strict) order preserving map on $P$ then this action induces isomorphic representations of $G$ on the $j$-th reduced simplicial homology $\widetilde{H}_{j}(P)$ and cohomology $\widetilde{H}^{j}(P)$ of the order complex $\Delta(P)$. Let $P$ be bounded (it has a minimal element (denoted $\hat{0}$ ) and a maximal element (denoted $\hat{1})$ ) and pure (all the maximal chains have the same length), if for any open interval $(x, y)$ in $P$ it happens that $\widetilde{H}_{i}((x, y))=0$ for all $x<y$ in $P$ and $i<l([x, y])-2$ we say that $P$ is Cohen-Macaulay. Some poset topology techniques on pure bounded posets, like the theory of lexicographic shellability, imply Cohen-Macaulayness.

Recall that the boolean algebra $\mathbb{B}_{n}$ is the poset of subsets of $[n]$ ordered by inclusion. It is known that $\mathbb{B}_{n}$ is Cohen-Macaulay and there is a natural action of $\mathfrak{S}_{n}$ on $\mathbb{B}_{n}$ permuting the elements in $[n]$. This action induces a representation of $\mathfrak{S}_{n}$ on the unique nonvanishing reduced simplicial cohomology $\widetilde{H}^{n-2}\left(\overline{\mathbb{B}}_{n}\right)$ of the proper part $\overline{\mathbb{B}}_{n}:=\mathbb{B}_{n} \backslash\{\hat{0}, \hat{1}\}$ of $\mathbb{B}_{n}$. The following isomorphism of $\mathfrak{S}_{n}$-representations is already a classical result (see [11]),

$$
\widetilde{H}^{n-2}\left(\overline{\mathbb{B}}_{n}\right) \simeq_{\mathfrak{S}_{n}} \operatorname{sgn}_{n} .
$$

\subsection{The weighted boolean algebra}

For undefined poset notation and terminology the reader can consult [14]. Let $\mathbb{W} \mathbb{C O M P}$ be the partially ordered set of weak compositions with order relation defined as follows: for every $\nu, \mu \in$ wcomp, we say that $\mu \leq \nu$ if $\mu(i) \leq \nu(i)$ for every $i$. We define $\mathbb{W} \mathbb{C O M P}$ to be the induced subposet of $\mathbb{W} \mathbb{C O M P}$ whose elements are weak compositions $\mu \in$ wcomp with $|\mu| \leq n$. A weighted subset of $[n]$ is a set $B^{\mu}$ where $B \subseteq[n]$ and $\mu \in$ wcomp $_{|B|}$. Since weak compositions are infinite vectors we can use componentwise addition and subtraction, for instance, we denote by $\nu+\mu$, the weak composition defined by $(\nu+$ $\mu)(i):=\nu(i)+\mu(i)$.

The weighted boolean algebra $\mathbb{B}_{n}^{w}$ is the partially ordered set (poset) of weighted subsets of $[n]$ with covering order relation given by $A^{\mu} \lessdot B^{\nu}$ if the following conditions hold:

- $A \lessdot B$ in $\mathbb{B}_{n}$ and,

- $\nu-\mu=\mathbf{e}_{\mathbf{r}}$ for some $r \in \mathbb{P}$, where $\mathbf{e}_{\mathbf{r}}$ is the weak composition with a 1 in the $r$-th component and 0 in all other entries.

For $S \in \mathbb{P}$ we also denote by $\mathbb{B}_{n}^{S}$ the induced subposet of $\mathbb{B}_{n}^{w}$ whose elements are weighted subsets $B^{\mu}$ with $\operatorname{supp}(\mu) \subseteq S$. In Figure $1\left(\right.$ a) we illustrate the Hasse diagram of the poset $\mathbb{B}_{3}^{[2]}$.

The poset $\mathbb{B}_{n}^{w}$ has a minimum element $\hat{0}:=\varnothing^{0}$ and maximal elements $[n]^{\mu}:=\left\{[n]^{\mu}\right\}$ indexed by weak compositions $\mu \in \mathrm{wcomp}_{n}$. Note that for every $\nu, \mu \in \mathrm{wcomp}_{n}$ such that $\nu$ is a rearrangement of $\mu$, the maximal intervals $\left[\hat{0},[n]^{\nu}\right]$ and $\left[\hat{0},[n]^{\mu}\right]$ are isomorphic to each other. In particular, if $\mu$ has a single nonzero component, these intervals are isomorphic to $\mathbb{B}_{n}$, hence $\mathbb{B}_{n}^{[1]} \simeq \mathbb{B}_{n}$. In the case when $S=[2]$ the poset $B_{n}^{[2]}$ is isomorphic to a poset introduced by Shareshian and Wachs in [10] and it is closely related to a poset of Björner and Welker in [2].

The symmetric group $\mathfrak{S}_{n}$ acts on $\mathbb{B}_{n}^{w}$. Indeed for any $B^{\mu} \in \mathbb{B}_{n}^{w}$ and $\tau \in \mathfrak{S}_{n}$ we have $\tau B^{\mu}:=(\tau B)^{\mu}$ where $\tau B:=\{\tau(i) \mid i \in B\}$. Since any $\tau \in \mathfrak{S}_{n}$ is a strict order preserving morphism, the action of 


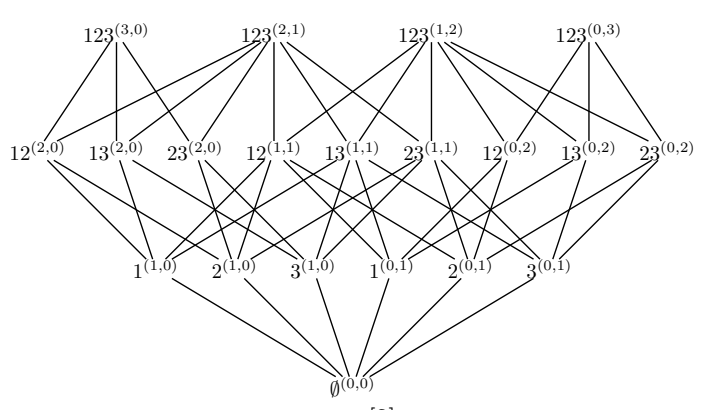

(a) $\mathbb{B}_{3}^{[2]}$

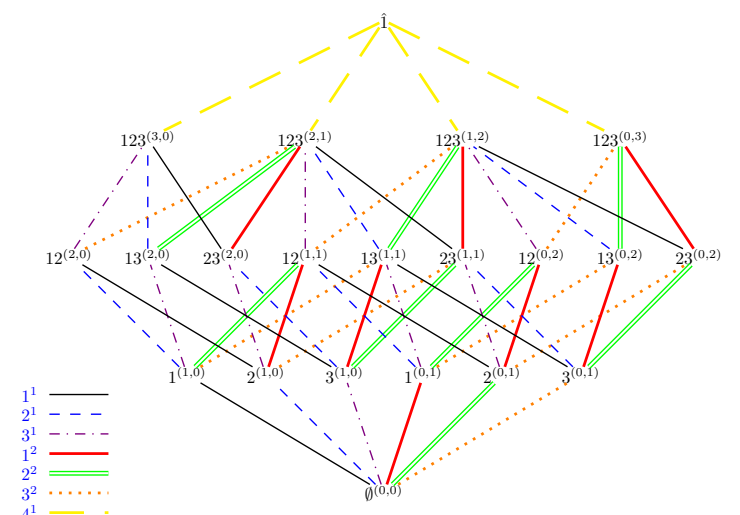

(b) A labeling of $\widehat{\mathbb{B}_{3}^{[2]}}$

Fig. 1: The weighted boolean algebra

$\mathfrak{S}_{n}$ on $\mathbb{B}_{n}^{w}$ induces and action on the unique reduced (co)homology $\widetilde{H}^{n-2}\left(\left(\hat{0},[n]^{\mu}\right)\right)$ of the open maximal interval $\left(\hat{0},[n]^{\mu}\right)$ of $\mathbb{B}_{n}^{w}$.

The outline of this extended abstract is as follows: In Section 2, for every $\mu \in$ wcomp $_{n}$ we give a generalization of the isomorphism (1.7). In Section 3 we use the more general isomorphism and an ELlabeling of $\widehat{\mathbb{B}_{n}^{w}}:=\mathbb{B}_{n}^{w} \cup\{\hat{1}\}$, the poset $\mathbb{B}_{n}^{w}$ after a maximal element has been added, to derive bases and compute the dimensions for $\Lambda(\mu)$ and $\widetilde{H}^{n-2}\left(\left(\hat{0},[n]^{\mu}\right)\right)$. In Section 4 we present a multiplicative inverse formula for the generating function of the Frobenius characteristic of $\Lambda(\mu)$ and $\widetilde{H}^{n-2}\left(\left(\hat{0},[n]^{\mu}\right)\right)$. This formula is derived in the full version of the paper using a technique of Sundaram [12] to compute group representations on Cohen-Macaulay $G$-posets. Then using the multiplicative inverse formula and a result of Gessel we describe explicitly the expansion of $\Lambda(\mu)$ and $\widetilde{H}^{n-2}\left(\left(\hat{0},[n]^{\mu}\right)\right)$ into irreducible representations. Finally, we use a technique of Méndez in Section 5 to conclude in Theorem 5.2 that for a finite $S$ and finite dimensional $V$ the Koszul dual algebras $\mathcal{S}_{S}(V)$ and $\Lambda_{S}(V)$ are Koszul in the sense of Priddy [9].

\section{The isomorphism}

\subsection{A generating set for $\widetilde{H}^{n-2}\left(\left(\hat{0},[n]^{\mu}\right)\right)$}

The top dimensional cohomology of a pure poset $P$, say of length $\ell$, has a particularly simple description. Let $\mathcal{M}(P)$ denote the set of maximal chains of $P$ and let $\mathcal{M}^{\prime}(P)$ denote the set of chains of length $\ell-1$. We view the coboundary map $\delta$ as a map from the chain space of $P$ to itself, which takes chains of length $r$ to chains of length $r+1$ for all $r$. Since the image of $\delta$ on the top chain space (i.e. the space spanned by $\mathcal{M}(P)$ ) is 0 , the kernel is the entire top chain space. Hence the top cohomology is the quotient of the space spanned by $\mathcal{M}(P)$ by the image of the space spanned by $\mathcal{M}^{\prime}(P)$. The image of $\mathcal{M}^{\prime}(P)$ is what we call the coboundary relations. We thus have as a presentation of the top cohomology

$$
\left.\widetilde{H}^{\ell}(P)=\langle\mathcal{M}(P)| \text { coboundary relations }\right\rangle \text {. }
$$


It can be shown (see for example the appendix in [5]) that the cohomology relations in the top cohomology are generated by relations of the form

$$
\sum_{\alpha \in\left(\alpha_{i-1}, \alpha_{i}\right)}\left(\alpha_{0}<\cdots<\alpha_{i-1}<\alpha<\alpha_{i}<\cdots<\alpha_{d}\right)=0
$$

where $\alpha_{0}<\cdots<\alpha_{\ell-1} \in \mathcal{M}^{\prime}(P)$ is a chain that can be refined (or augmented) in exactly one step $\alpha_{i-1}<\alpha_{i}$ for some $i=0, \ldots, \ell$ to get a chain in $\mathcal{M}(P)$.

It is not too hard to see that the maximal chains in a maximal interval $\left[\hat{0},[n]^{\mu}\right]$ of $\mathbb{B}_{n}^{w}$ are in bijection with colored permutations. Indeed, a map $\bar{\lambda}: \mathcal{E}(P) \rightarrow \Lambda$, where $\mathcal{E}(P)$ is the set of edges (covering relations) of the Hasse diagram of a poset $P$ and $\Lambda$ is a fixed poset is called an edge labeling. Note that a covering relation in $\mathbb{B}_{n}^{w}$ is of the form $A^{\nu} \lessdot(A \cup\{x\})^{a+\mathbf{e}_{\mathbf{i}}}$ where $A^{\nu}$ is a weighted subset of $[n], x \in[n] \backslash A$ and $i \in \mathbb{P}$. So we can associate a labeling $\bar{\lambda}: \mathcal{E}\left(\mathbb{B}_{n}^{w}\right) \rightarrow[n]^{\mathbb{P}}$ given by

$$
\bar{\lambda}\left(A^{\nu},(A \cup\{x\})^{\nu+\mathbf{e}_{\mathbf{i}}}\right)=x^{i} .
$$

We will show in Section 3 that this labeling $\bar{\lambda}$ can be extended to an EL-labeling (defined in Section 3.1) of $\widehat{\mathbb{B}_{n}^{w}}:=\mathbb{B}_{n}^{w} \cup\{\hat{1}\}$, that is $\mathbb{B}_{n}^{w}$ with a maximal element added (See also Figure 1(b)). We denote by

$$
\bar{\lambda}(\mathrm{c})=\bar{\lambda}\left(x_{0}, x_{1}\right) \bar{\lambda}\left(x_{1}, x_{2}\right) \cdots \bar{\lambda}\left(x_{\ell-1}, x_{\ell}\right),
$$

the word of labels corresponding to a maximal chain $\mathrm{c}=\left(\hat{0}=x_{0} \lessdot x_{1} \lessdot \cdots \lessdot x_{\ell-1} \lessdot x_{\ell}=\hat{1}\right)$. In the case $\mathrm{c} \in \mathcal{M}\left(\left[\hat{0},[n]^{\mu}\right]\right)$ it is immediate that $\bar{\lambda}(\mathrm{c}) \in \mathfrak{S}_{\mu}$ since in each covering relation in $\mathrm{c}$ a different letter from $[n]$ appears in each step and $\mathrm{c}$ has length $n$. Moreover, c follows the sequence of colors corresponding to $\mu$. For example the chain

$$
\hat{0} \lessdot\{2\}^{(1,0,0,0)} \lessdot\{1,2\}^{(1,0,0,1)} \lessdot\{1,2,3\}^{(1,1,0,1)}
$$

has the colored permutation $2^{1} 1^{4} 3^{2}$ as a word of labels. Clearly, starting with $\sigma \in \mathfrak{S}_{\mu}$ we can also recover the chain $\mathrm{c} \in \mathcal{M}\left(\left[\hat{0},[n]^{\mu}\right]\right)$ such that $\bar{\lambda}(\mathrm{c})=\sigma$. Indeed, for $\sigma \in \mathfrak{S}_{\mu}$ define the chain $\mathrm{c}(\sigma) \in \mathcal{M}\left(\left[\hat{0},[n]^{\mu}\right]\right)$ to be the one whose rank 0 element is $\hat{0}$ and whose rank $i$ weighted subset is

$$
\{\sigma(1), \sigma(2), \ldots, \sigma(i)\}^{\mathbf{e}_{\operatorname{color}(\sigma(1))}+\mathbf{e}_{\mathbf{c o l o r}(\sigma(2))}+\cdots+\mathbf{e}_{\operatorname{color}(\sigma(\mathbf{i}))}}
$$

for all $i \in[n]$. Using induction we can conclude the following theorem.

Proposition 2.1 The maps $\bar{\lambda}$ and $\mathrm{c}$ above define a bijection

$$
\mathcal{M}\left(\left[\hat{0},[n]^{\mu}\right]\right) \simeq \mathfrak{S}_{\mu} .
$$

Note also that for a bounded poset $P$ the sets $\mathcal{M}(P)$ and $\mathcal{M}(P \backslash\{\hat{0}, \hat{1}\})$ are in bijection by associating a chain $\mathrm{c} \in \mathcal{M}(P)$ with a chain $\overline{\mathrm{c}}:=\mathrm{c} \backslash\{\hat{0}, \hat{1}\} \in \mathcal{M}(P \backslash\{\hat{0}, \hat{1}\})$. For $\sigma \in \mathfrak{S}_{\mu}$ we write $\overline{\mathrm{c}}(\sigma):=c(\sigma) \backslash\{\hat{0}, \hat{1}\}$ for the corresponding chain in $\left(\hat{0},[n]^{\mu}\right)$.

The codimension one cohomology relations $[2.1)$ in $\left(\hat{0},[n]^{\mu}\right)$ correspond to the two different types of intervals of length 2 in $\left[\hat{0},[n]^{\mu}\right]$. The intervals of length 2 happen when two elements $x$ and $y$ have been added to a weighted subset $A^{a}$ and the weight has been increased accordingly. The Type $I$ intervals occur when the weight has been increased by $2 \mathrm{e}_{\mathrm{i}}$ and the Type II intervals when the weight has been increased by $\mathbf{e}_{\mathbf{i}}+\mathbf{e}_{\mathbf{j}}$ with $i \neq j \in \mathbb{P}$. In the following we denote $\alpha x^{i} y^{i} \beta$ to a colored permutation where $\alpha$ and $\beta$ denote the starting and trailing colored subwords. 
Theorem 2.2 The set $\left\{\overline{\mathrm{c}}(\sigma) \mid \sigma \in \mathfrak{S}_{\mu}\right\}$ is a generating set for $\widetilde{H}^{n-2}\left(\left(\hat{0},[n]^{\mu}\right)\right)$, subject only to the relations for $i \neq j \in \operatorname{supp}(\mu)$

$$
\begin{aligned}
& \overline{\mathrm{c}}\left(\alpha x^{i} y^{i} \beta\right)+\overline{\mathrm{c}}\left(\alpha y^{i} x^{i} \beta\right)=0 \\
& \overline{\mathrm{c}}\left(\alpha x^{i} y^{j} \beta\right)+\overline{\mathrm{c}}\left(\alpha y^{j} x^{i} \beta\right)+\overline{\mathrm{c}}\left(\alpha y^{i} x^{j} \beta\right)+\overline{\mathrm{c}}\left(\alpha x^{j} y^{i} \beta\right)=0
\end{aligned}
$$

Proof: Observe that the relations (2.3) and 2.4 correspond exactly to the codimension one cohomology relations of Type I and Type II respectively.

\subsection{The isomorphism}

Note that following the relations (1.5) and 1.6) we can conclude a similar proposition for $\Lambda(\mu)$.

Proposition 2.3 The set $\left\{\wedge(\sigma) \mid \sigma \in \mathfrak{S}_{\mu}\right\}$ is a generating set for $\Lambda(\mu)$ subject only to the relations for $i \neq j \in \operatorname{supp}(\mu)$

$$
\begin{aligned}
& \wedge\left(\alpha x^{i} y^{i} \beta\right)+\wedge\left(\alpha y^{i} x^{i} \beta\right)=0 \\
& \wedge\left(\alpha x^{i} y^{j} \beta\right)+\wedge\left(\alpha y^{j} x^{i} \beta\right)+\wedge\left(\alpha y^{i} x^{j} \beta\right)+\wedge\left(\alpha x^{j} y^{i} \beta\right)=0
\end{aligned}
$$

Theorem 2.4 For each $\mu \in \mathrm{wcomp}_{n}$, the map $\varphi: \Lambda(\mu) \rightarrow \widetilde{H}^{n-2}\left(\left(\hat{0},[n]^{\mu}\right)\right)$ determined by

$$
\varphi(\wedge(\sigma))=\overline{\mathrm{c}}(\sigma) \quad \text { for all } \sigma \in \mathfrak{S}_{\mu},
$$

is an $\mathfrak{S}_{n}$-module isomorphism.

Proof: The generators of the two sets $\Lambda(\mu)$ and $\widetilde{H}^{n-2}\left(\left(\hat{0},[n]^{\mu}\right)\right)$ are indexed by colored permutations in $\mathfrak{S}_{\mu}$ and $\varphi$ maps generators to generators. By Theorem 2.2 and Proposition $2.3 \varphi$ also maps relations to relations and clearly respects the $\mathfrak{S}_{n}$-action.

\section{Homotopy type of maximal intervals in $\mathbb{B}_{n}^{w}$}

\subsection{EL-labeling}

Let $P$ be a bounded poset. Recall the definition of edge labeling from Section 2 . We say that a maximal chain $c$ is increasing if its word of labels $\bar{\lambda}(c)$ is strictly increasing, that is, $c$ is increasing if

$$
\bar{\lambda}\left(x_{0}, x_{1}\right)<\bar{\lambda}\left(x_{1}, x_{2}\right)<\cdots<\bar{\lambda}\left(x_{\ell-1}, x_{\ell}\right) .
$$

We say that $c$ is ascent-free (or decreasing, or falling) if its word of labels $\bar{\lambda}(c)$ has no ascents, i.e. $\bar{\lambda}\left(x_{i}, x_{i+1}\right) \nless \bar{\lambda}\left(x_{i+1}, x_{i+2}\right)$, for all $i=0, \ldots, t-2$. An edge-lexicographical labeling (EL-labeling, for short) of $P$ is an edge labeling such that in each closed interval $[x, y]$ of $P$, there is a unique increasing maximal chain, and this chain lexicographically precedes all other maximal chains of $[x, y]$. See [1] for more information about EL-labelings.

We let $\Lambda=[n+1]^{\mathbb{P}}:=[n+1] \times \mathbb{P}$ the product poset of the totally ordered sets $[n+1]$ and $\mathbb{P}$ and we define for any $S \subseteq \mathbb{P}$ the labeling $\bar{\lambda}: \mathcal{E}\left(\widehat{\mathbb{B}_{n}^{S}}\right) \rightarrow[n+1]^{\mathbb{P}}$ by

$$
\bar{\lambda}\left(A^{\nu},(A \cup\{x\})^{\nu+\mathbf{e}_{\mathbf{i}}}\right)=x^{i} \quad \text { and } \quad \bar{\lambda}\left([n]^{\mu}, \hat{1}\right)=(n+1)^{1} .
$$


Theorem 3.1 The labeling $\bar{\lambda}: \mathcal{E}\left(\widehat{\mathbb{B}_{n}^{S}}\right) \rightarrow[n+1]^{\mathbb{P}}$ in $\sqrt{3.1}$ is an EL-labeling of $\widehat{\mathbb{B}_{n}^{S}}$.

The proof of this theorem is similar to the one of [5, Theorem 3.2] and is given in the full version of this paper. In Figure 1(b) the labeling of $\widehat{\mathbb{B}_{n}^{[2]}}$ is illustrated. The edges have been differentiated by color corresponding to the different labels in the legend.

The following theorem links lexicographic shellability of a poset $P$ with the topology of $\Delta(P)$.

Theorem 3.2 (Björner and Wachs [1]) Let $\bar{\lambda}$ be an EL-labeling of a bounded poset P. Then $\Delta(\bar{P})$ is homotopy equivalent to a wedge of spheres of dimension $\ell(P)-2$, where the number of spheres is the number of ascent-free maximal chains in $P$. Moreover the set $\{\bar{c} \in \mathcal{M}(P) \mid c$ is ascent-free $\}$ forms a basis for the cohomology $\widetilde{H}^{\ell(P)-2}(\bar{P})$.

We would like to characterize the ascent-free chains of the EL-labeling of Theorem 3.1 We already know by Proposition 2.1 that the maximal chains in $\mathbb{B}_{n}^{S}$ are in bijection with permutations in $\mathfrak{S}_{n}^{S}$. Since any maximal chain in $\widehat{\mathbb{B}_{n}^{S}}$ is of the form $\mathrm{c}^{\prime} \cup\{\hat{1}\}$ where $\mathrm{c}^{\prime}$ is a maximal chain in $\mathbb{B}_{n}^{S}$ then the permutations in $\mathfrak{S}_{n}^{S}$ are also in bijection with maximal chains in $\widehat{\mathbb{B}_{n}^{S}}$. For $\sigma \in \mathfrak{S}_{n}^{S}$ denote $\hat{c}(\sigma):=c(\sigma) \cup\{\hat{1}\}$.

An ascent in a colored permutation $\sigma \in \mathfrak{S}_{n}^{\mathbb{P}}$ is a value $i \in[n-1]$ such that

- $\sigma(i)<\sigma(i+1)$, that is, $i$ is an ascent in the underlying uncolored permutation $\tilde{\sigma} \in \mathfrak{S}_{n}$, and

- $\operatorname{color}(\sigma(i)) \leq \operatorname{color}(\sigma(i+1))$.

A nonincreasing colored permutation is a colored permutation $\sigma \in \mathfrak{S}_{n}^{\mathbb{P}}$ that is ascent free. For example $2^{1} 1^{4} 3^{2}$ is a nonincreasing colored permutation but $2^{1} 1^{2} 3^{4}$ is not since the pair $\left(1^{2}, 3^{4}\right)$ forms an ascent. Let Ninc ${ }_{n}$ be the set of nonincreasing colored permutations and $\mathrm{Ninc}_{\mu}$ the ones with content $\mu$. Moreover, denote by $\overline{\operatorname{Ninc}_{n}^{S}}$ the set of permutations in $\operatorname{Ninc}_{n}^{S}$ with $\operatorname{color}(\sigma(n)) \neq 1$. We have the following characterization of the ascent-free chains.

Theorem 3.3 The set $\left\{\hat{\mathrm{c}}(\sigma) \mid \sigma \in \overline{\operatorname{Ninc}_{n}^{S}}\right\}$ is the set of ascent-free maximal chains of $\widehat{\mathbb{B}_{n}^{S}}$ and for every $\mu \in$ wcomp, the set $\left\{\mathrm{c}(\sigma) \mid \sigma \in\right.$ Ninc $\left._{\mu}\right\}$ is the set of ascent-free maximal chains of $\left[\hat{0},[n]^{\mu}\right]$ in the ELlabeling of Theorem 3.1. Consequently, the poset $\widehat{\mathbb{B}_{n}^{S}}$ is Cohen-Macaulay and its order complex $\Delta\left(\mathbb{B}_{n}^{S} \backslash\right.$ $\{\hat{0}\})$ has the homotopy type of a wedge of $\left|\overline{\operatorname{Ninc}_{n}^{S}}\right|$ spheres of dimension $(n-1)$ and the interval $\left[\hat{0},[n]^{\mu}\right]$ is Cohen-Macaulay and its order complex $\Delta\left(\left(\hat{0},[n]^{\mu}\right)\right)$ has the homotopy type of a wedge of $\mid$ Ninc $_{\mu} \mid$ spheres of dimension $(n-2)$.

Corollary 3.4 The set $\left\{\mathrm{c}(\sigma) \backslash\{\hat{0}\} \mid \sigma \in \overline{\operatorname{Ninc}_{n}^{S}}\right\}$ is a basis for $\widetilde{H}^{n-1}\left(\mathbb{B}_{n}^{S} \backslash\{\hat{0}\}\right)$ and for every $\mu \in$ wcomp the set $\left\{\overline{\mathrm{c}}(\sigma) \mid \sigma \in\right.$ Ninc $\left._{\mu}\right\}$ is a basis for $\widetilde{H}^{n-2}\left(\left(\hat{0},[n]^{\mu}\right)\right)$.

Using the isomorphism of Theorem 2.4 and Corollary 3.4 we conclude the following theorem.

Theorem 3.5 For $\mu \in$ wcomp, the set $\left\{\wedge(\sigma) \mid \sigma \in\right.$ Ninc $\left._{\mu}\right\}$ is a basis for $\Lambda(\mu)$. Consequently,

$$
\operatorname{dim} \Lambda(\mu)=\mid \text { Ninc }_{\mu} \mid \text {. }
$$

Consider the generating function

$$
\sum_{\mu \in \text { wcomp }_{n}} \operatorname{dim} \Lambda(\mu) \mathbf{x}^{\mu}
$$


where $\mathbf{x}^{\mu}=x_{1}^{\mu(1)} x_{2}^{\mu(2)} \ldots$. Since $\operatorname{dim} \Lambda(\mu)$ is invariant under any rearrangement of the parts of $\mu$ we have that 3.2 belongs to the ring of symmetric functions $\Lambda_{\mathbb{Z}}$. The symmetric function (3.2) is also $e$-positive; i.e., its coefficients of the expansion in the basis of elementary symmetric functions are all nonnegative. Indeed, we associate a type (or integer partition) to each $\sigma \in \mathfrak{S}_{n}$ in the following way: Let $\pi(\sigma)$ be the finest (set) partition of the set $[n]$ satisfying

- whenever $\sigma(i)<\sigma(i+1)$ for some $i \in[n-1], \sigma(i)$ and $\sigma(i+1)$ belong to the same block of $\pi(\sigma)$.

We define the type $\lambda(\sigma)$ of $\sigma$ to be the (integer) partition whose parts are the sizes of the blocks of $\pi(\sigma)$. For example, for the permutation 5126473 the associated partition is $\lambda(\sigma)=(3,2,1,1)$.

Theorem 3.6 For all $n$,

$$
\sum_{\mu \in \text { wcomp }_{n}} \operatorname{dim} \Lambda(\mu) \mathbf{x}^{\mu}=\sum_{\sigma \in \mathfrak{S}_{n}} e_{\lambda(\sigma)}(\mathbf{x})
$$

where $e_{\lambda}$ is the elementary symmetric function associated with the partition $\lambda$.

Proof: If $\sigma \in \mathfrak{S}_{n}^{\mathbb{P}}$ is a colored permutation, denote by $\tilde{\sigma} \in \mathfrak{S}_{n}$ the underlying uncolored permutation associated to $\sigma$. For example if $\sigma=2^{1} 1^{4} 3^{2}$ then $\tilde{\sigma}=213$. Note that the type $\lambda(\tau)$ for a permutation $\tau \in \mathfrak{S}_{n}$ is closely related to the coloring condition in Ninc N $_{n}$. If $\sigma \in$ Ninc $_{n}$ such that $\tilde{\sigma}=\tau$ the colors in each part of the partition $\pi(\tau)$ need to strictly decrease from left to right. If $B$ is a block of $\pi(\tau)$ of size $|B|=i$ then the elementary symmetric function $e_{i}(\mathbf{x})$ enumerates all the possible ways of coloring the letters in $B$. Then the contribution to the generating function (3.2) of all the nonincreasing colored permutations with underlying uncolored permutation $\tau$ is $e_{\lambda(\tau)}$.

Theorem 4.3 in Section 4 provides another characterization of the symmetric function 3.2.

\section{The Frobenius characteristic of $\Lambda(\mu)$}

\subsection{A multiplicative inverse formula}

For some ring $R$, let $\Lambda_{R}$ denote the ring of symmetric functions with coefficients in $R$ and $\widehat{\Lambda_{R}}$ the completion of $\Lambda_{R}$ with respect to the Hall's inner product (i.e., the ring of symmetric power series with coefficients in $R$ ). It is known that the Grothendieck ring of representations of symmetric groups $R e p_{\mathfrak{S}}$ is isomorphic to the ring of symmetric functions $\Lambda_{\mathbb{Z}}$ (we use here variables $\mathbf{y}=\left(y_{1}, y_{2}, \ldots\right)$ ) under the Frobenius characteristic map ch $: \operatorname{Rep}_{\mathfrak{S}} \rightarrow \Lambda_{\mathbb{Z}}$. Under this isomorphism, for a (perhaps empty) integer partition $\lambda$ the Specht module $S^{\lambda}$ indexed by $\lambda$ maps to the Schur function $s_{\lambda}$.

Theorem 4.1 We have that

$$
\sum_{n \geq 0} \sum_{\mu \in \mathrm{wcomp}_{n}} \operatorname{ch} \widetilde{H}^{n-2}\left(\left(\hat{0},[n]^{\mu}\right)\right) \mathbf{x}^{\mu}=\left(\sum_{n \geq 0}(-1)^{n} h_{n}(\mathbf{x}) h_{n}(\mathbf{y})\right)^{-1}
$$

where $h_{n}$ is the complete homogeneous symmetric function and $(\cdot)^{-1}$ denotes the multiplicative inverse of a formal power series. 
The proof of Theorem 4.1 in the full version of this paper requires various technical lemmas and uses a technique introduced by Sundaram [12] to compute group representations on the (co)homology of CohenMacaulay $G$-posets (see [4] for the proof of an analogous result).

The following theorem is a corollary of the isomorphism of Theorem 2.4 and of Theorem 4.1

Theorem 4.2 We have that

$$
\sum_{n \geq 0} \sum_{\mu \in \text { wcomp }_{n}} \operatorname{ch} \Lambda(\mu) \mathbf{x}^{\mu}=\left(\sum_{n \geq 0}(-1)^{n} h_{n}(\mathbf{x}) h_{n}(\mathbf{y})\right)^{-1} .
$$

Note that the power series in Theorems 4.1 and 4.2 belong to $\widehat{\Lambda_{R}}$ with $R=\Lambda_{\mathbb{Q}}$. Now consider the map $E: \widehat{\Lambda_{R}} \rightarrow R[[y]]$ defined by $E\left(p_{i}(\mathbf{y})\right)=y \delta_{i, 1}$ for $i \geq 1$ and extended multiplicatively, linearly and taking the corresponding limits to all of $\widehat{\Lambda_{R}}$. Note that $E$ is a ring homomorphism since $E$ is defined on generators. The effect of $E$ can be better understood under the following definition of the Frobenius map.

$$
E(\operatorname{ch} V)=E\left(\frac{1}{n !} \sum_{\sigma \in \mathfrak{S}_{n}} \chi^{V}(\sigma) p_{\lambda(\sigma)}(\mathbf{y})\right)=\frac{1}{n !} \sum_{\sigma \in \mathfrak{S}_{n}} \chi^{V}(\sigma) E\left(p_{\lambda(\sigma)}(\mathbf{y})\right)=\chi_{V}(i d) \frac{y^{n}}{n !}=\operatorname{dim} V \frac{y^{n}}{n !},
$$

where $V$ is a representation of $\mathfrak{S}_{n}, \chi^{V}$ its character and $\lambda(\sigma)$ is the cycle type of $\sigma \in \mathfrak{S}_{n}$. In particular since $h_{n}(\mathbf{y})=\operatorname{ch}\left(\mathbf{1}_{\mathbf{n}}\right)$, the Frobenius characteristic of the trivial representation, we have that $E\left(h_{n}(\mathbf{y})\right)=$ $y^{n} / n$ !. Applying $E$ in Theorem 4.2 yields another characterization of the family of symmetric functions 3.2.

Theorem 4.3 We have

$$
\sum_{n \geq 0} \sum_{\mu \in \text { wcomp }_{n-1}} \operatorname{dim} \Lambda(\mu) \mathbf{x}^{\mu} \frac{y^{n}}{n !}=\left[\sum_{n \geq 0}(-1)^{n} h_{n}(\mathbf{x}) \frac{y^{n}}{n !}\right]^{-1} .
$$

Theorem 4.3 can be also proven directly using for example the recursive definition of the Möbius invariant of the maximal intervals of $\mathbb{B}_{n}^{w}$, Philip Hall's theorem (see for example [14]) and Theorem 2.4

\subsection{Explicit description of the $\mathfrak{S}_{n}$ representation on $\Lambda(n)$}

A composition $\alpha=\left(\alpha_{1}, \ldots, \alpha_{\ell}\right)$ is a finite sequence of elements $\alpha_{i} \in \mathbb{P}$. We say that $\alpha$ is a composition of $n$ if $|\alpha|:=\sum_{i} \alpha_{i}=n$ and we denote by $\operatorname{comp}_{n}$ the set of compositions of $n$. We denote $\lambda(\alpha)$ the integer partition obtained from $\alpha$ by reordering its parts in weakly decreasing order. A skew hook is a connected skew shape that avoids the shape $(2,2)$. Every skew hook can be descrided by a composition $\alpha$ whose parts are the lengths of the horizontal steps from left to right. We denote by $H(\alpha)$ the skew hook determined by $\alpha \in$ comp. See Figure 2 for an example of a skew hook.

In the full version of this article we give a proof of the following theorem using the combinatorial interpretation of the multiplicative inverse of an ordinary generating function in terms of words with allowed and forbidden links discovered by Carlitz-Scoville-Vaughan [3].

Theorem 4.4 (Gessel, personal communication) We have that

$$
\left(\sum_{n \geq 0}(-1)^{n} h_{n}(\mathbf{x}) h_{n}(\mathbf{y})\right)^{-1}=\sum_{n \geq 0} \sum_{\alpha \in \operatorname{comp}_{n}} e_{\lambda(\alpha)}(\mathbf{x}) s_{H(\alpha)}(\mathbf{y}) .
$$




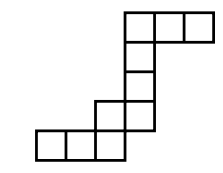

Fig. 2: Skew hook $H(3,2,1,1,3)$

If we number the cells of a skew hook $H$ left-to-right and bottom-to-top, $i$ is a descent of $H$ if the cell $i+1$ is above the cell $i$. We denote by $\operatorname{des}(H)$ the descent set of $H$. For a standard Young tableau of shape $\lambda$ a descent is an entry $i$ that is in a higher row than $i+1$.

Proposition 4.5 (c.f. [14]) For a skew hook $H$ we have that

$$
s_{H}(\mathbf{y})=\sum_{\lambda \vdash n} c_{H, \lambda} s_{\lambda}(\mathbf{y}),
$$

where $c_{H, \lambda}$ is the number of Young tableaux of shape $\lambda$ and descent set $\operatorname{des}(H)$.

Theorems 4.3 and 4.4 together with Proposition 4.5 yield Theorem 4.6 as a corollary.

Theorem 4.6 For $n \geq 1$ we have that

$$
\sum_{\mu \in \mathrm{wcomp}_{n}} \operatorname{ch} \Lambda(\mu) \mathbf{x}^{\mu}=\sum_{\eta \vdash n} \sum_{\alpha \in \operatorname{comp}_{n}} c_{H(\alpha), \eta} e_{\lambda(\alpha)}(\mathbf{x}) s_{\eta}(\mathbf{y}) .
$$

Theorem 4.6 can be seen as an equivariant version of the $e$-positivity in Theorem 3.6 Indeed, if we write

$$
\sum_{\mu \in \mathrm{wcomp}_{n}} \operatorname{ch} \Lambda(\mu) \mathbf{x}^{\mu}=\sum_{\lambda \vdash n} C_{\lambda}(\mathbf{y}) e_{\lambda}(\mathbf{x}),
$$

then Theorem 4.6 implies that the coefficients $C_{\lambda}(\mathbf{y})$ are Schur-positive.

\section{The Koszul property}

A quadratic associative algebra $A$ and its Koszul dual (co)algebra $A^{\mathrm{i}}$ are said to be Koszul if the Koszul complex $A^{\mathrm{i}} \otimes_{\kappa} A$ is acyclic (see [7] for the definitions). There are various techniques to conclude the Koszul property of an associative algebra. We use the technique in [8] that involves determining that a family of posets associated to certain types of algebras are Cohen-Macaulay. For a finite subset $S \in \mathbb{P}$ the equivalence classes of generators, after the symmetry relations (1.3) and (1.4), in $\mathcal{S}_{S}(n)$ can be identified with colored subsets $A^{\mu}$, where $A \subseteq[n]$ and $\mu \in$ wcomp $_{|A|}$ with $\operatorname{supp}(\mu) \in S$. Let $\overline{\mathcal{S}}_{S}(n)$ be the set of colored subsets whose underlying set is $A=[n]$, the map $[n] \mapsto \overline{\mathcal{S}}_{S}(n)$ defines a functor (Joyal's species) from the category Set of finite sets and bijections to the category $\mathfrak{F}$ of finite sets and arbitrary functions. It is easy to verify that this functor is a quadratic (by relations (1.3) and (1.4) injective (cancellative) monoid or c-monoid in the sense of [8] and that the poset associated to $\overline{\mathcal{S}}_{S}$ in the construction in [8, Section $5.1]$ is precisely the family of posets $\mathbb{B}_{n}^{S}$ for $n \geq 0$. Moreover, the analytic functor $\mathcal{S}_{S}: V e c t_{\mathbf{k}} \rightarrow V e c t_{\mathbf{k}}$ associated to $\overline{\mathcal{S}}_{S}$ is $V \mapsto \mathcal{S}_{S}(V)$. 
Theorem 5.1 ([8, Proposition 22, Lemma 40, Theorem 41]) Let $\bar{M}$ be a c-monoid with associated analytic monoid $M$. Then $M$ and its Koszul dual $M^{!}$are Koszul if and only if the maximal intervals of the poset associated to $\bar{M}$ are Cohen-Macaulay.

Hence the fact that $\mathcal{S}_{S}(V)$ and $\Lambda_{S}(V)$ are Koszul is a consequence of Theorem 3.3 and 5.1 (We provide all the details in the full version of this paper).

Theorem 5.2 For a finite dimensional vector space $V$ and a finite subset $S \subseteq \mathbb{P}$, the Koszul dual associative algebras $\mathcal{S}_{S}(V)$ and $\Lambda_{S}(V)$ are Koszul.

\section{References}

[1] A. Björner and M. L. Wachs. On lexicographically shellable posets. Trans. Amer. Math. Soc., 277(1):323-341, 1983.

[2] A. Björner and V. Welker. Segre and Rees products of posets, with ring-theoretic applications. $J$. Pure Appl. Algebra, 198(1-3):43-55, 2005.

[3] L. Carlitz, R. Scoville, and T. Vaughan. Enumeration of pairs of sequences by rises, falls and levels. Manuscripta Math., 19(3):211-243, 1976.

[4] R. S. González D'León. On the free Lie algebra with multiple brackets. ArXiv e-prints, Aug. 2014.

[5] R. S. González D'León and M. L. Wachs. On the (co)homology of the poset of weighted partitions. Trans. Amer. Math. Soc., 368(10):6779-6818, 2016.

[6] P. Hanlon and M. L. Wachs. On Lie k-algebras. Adv. Math., 113(2):206-236, 1995.

[7] J.-L. Loday and B. Vallette. Algebraic operads, volume 346 of Grundlehren der Mathematischen Wissenschaften [Fundamental Principles of Mathematical Sciences]. Springer, Heidelberg, 2012.

[8] M. A. Méndez. Koszul duality for monoids and the operad of enriched rooted trees. Adv. in Appl. Math., 44(3):261-297, 2010.

[9] S. B. Priddy. Koszul resolutions. Trans. Amer. Math. Soc., 152:39-60, 1970.

[10] J. Shareshian and M. L. Wachs. Poset homology of Rees products, and $q$-Eulerian polynomials. Electron. J. Combin., 16(2, Special volume in honor of Anders Bjorner):Research Paper 20, 29, 2009.

[11] L. Solomon. A decomposition of the group algebra of a finite Coxeter group. J. Algebra, 9:220-239, 1968.

[12] S. Sundaram. The homology representations of the symmetric group on Cohen-Macaulay subposets of the partition lattice. Adv. Math., 104(2):225-296, 1994.

[13] M. L. Wachs. On the (co)homology of the partition lattice and the free Lie algebra. Discrete Math., 193(1-3):287-319, 1998. Selected papers in honor of Adriano Garsia (Taormina, 1994).

[14] M. L. Wachs. Poset topology: tools and applications. In Geometric combinatorics, volume 13 of IAS/Park City Math. Ser., pages 497-615. Amer. Math. Soc., Providence, RI, 2007. 